\title{
Croyances Traditionnelles et Conservation du Crocodylus Suchus Dans les Mares Sacrées de Bazoulé et de Sabou (Burkina Faso)
}

\author{
Ilassa Ouedraogo, \\ Adama Oueda, \\ Napoko Malika Kangoye, \\ Laboratoire de Biologie et Écologie Animales, \\ Université Joseph KI-ZERBO, Burkina Faso, Ouagadougou \\ Massamba Thiam, \\ Laboratoire de Zoologie des Vertébrés Terrestres, \\ Université Cheikh Anta Diop de Dakar Sénégal, Dakar \\ Justine Kabore, \\ Gustave B. Kabre, \\ Laboratoire de Biologie et Écologie Animales, \\ Université Joseph KI-ZERBO, Burkina Faso, Ouagadougou
}

Doi:10.19044/esj.2020.v16n6p188 URL:http://dx.doi.org/10.19044/esj.2020.v16n6p188

\section{Résumé}

De janvier 2016 à mai 2017, C. suchus de deux mares sacrées des villages de Bazoulé et de Sabou ont fait l'objet d'un suivi. L'objectif était de mettre en évidence les perceptions et les croyances locales des populations, puis capitaliser les mesures de conservation endogènes. Les attitudes, les croyances et les perceptions locales des populations sur le crocodile ont été étudiées à partir d'une approche ethno-zoologique basée sur des interviews à l'aide d'un questionnaire. Des inventaires diurnes et nocturnes ont été conduits afin de déterminer la structure de la population de crocodiles. Les résultats de cette étude suggèrent que les croyances traditionnelles jouent un rôle très important dans la protection et la conservation de C. suchus dans les villages de Bazoulé et Sabou. Un total de 268 individus de crocodiles a été dénombré à Bazoulé dont $25 \%$ de nouveau-nés ; $17 \%$ de juvéniles ; $12 \%$ de subadultes et $10 \%$ d'adultes. À Sabou sur un total de 249 individus dénombrés, $22 \%$ sont des nouveau-nés ; $12 \%$ des juvéniles ; $12 \%$ des subadultes et $14 \%$ des adultes. Malgré les perceptions et les croyances traditionnelles qui assurent une certaine protection des crocodiles dans ces mares, des pressions dues aux activités anthropiques pourraient influencer leur abondance. 
Mots clés : C. Suchus, Sites Sacrés, Activités Anthropiques, Burkina Faso

\title{
Traditional Beliefs and Conservation Crocodylus Suchus (Étienne Geoffroy Saint-Hilaire 1807) in the Sacred Ponds of Bazoule and Sabou (Burkina Faso)
}

Ilassa Ouedraogo,

Adama Oueda, Napoko Malika Kangoye,

Laboratoire de Biologie et Écologie Animales, Université Joseph KI-ZERBO, Burkina Faso, Ouagadougou

Massamba Thiam,

Laboratoire de Zoologie des Vertébrés Terrestres,

Université Cheikh Anta Diop de Dakar Sénégal, Dakar

Justine Kabore,

Gustave B. Kabre,

Laboratoire de Biologie et Écologie Animales, Université Joseph KI-ZERBO, Burkina Faso, Ouagadougou

\begin{abstract}
From January 2016 to May 2017, C. suchus from two sacred ponds in the villages of Bazoule and Sabou were monitored. The objective of this study was to highlight the local perceptions and beliefs of the populations, then capitalize on endogenous conservation measures. The local attitudes, beliefs and perceptions of the population about the crocodile were studied using an ethno-zoological approach based on interviews using a questionnaire. Diurnal and nocturnal inventories have been conducted to determine the crocodile population structure. The results of this study suggest that traditional beliefs play a very important role in the protection and conservation of $\mathrm{C}$. suchus in Bazoule and Sabou villages. A total of 268 crocodile individuals have been counted in Bazoule: $25 \%$ of hatchlings; $17 \%$ of juveniles; $12 \%$ of subadults and $10 \%$ of adults. In Sabou out of a total of 249 individuals counted, $22 \%$ are hatchlings; $12 \%$ juveniles; $12 \%$ of sub-adults and $14 \%$ of adults. Despite traditional perceptions and beliefs that provide some protection for crocodiles in these ponds, pressures from anthropogenic activities could influence their abundance.
\end{abstract}


Keywords: C. Suchus, Sacred Poods, Anthropogenic Activities, Burkina Faso

\section{Introduction}

La conservation et la protection de la biodiversité font actuellement partie des principaux objectifs de l'humanité. Elles sont devenues aujourd'hui une préoccupation majeure dans le monde (Hanski, 2005). La perte de la biodiversité est sans précédent et irréversible (Ceballos et al., 2015). Et l'une des causes principales de cette perte est l'élimination ou la dégradation des habitats naturels dans le monde (Krauss et al., 2010). Parmi cette biodiversité, les reptiles et en particulier le crocodile méritent une attention particulière vue le rôle important qu'il joue dans le fonctionnement et l'équilibre de l'écosystème aquatique et sur le plan touristique et cultuel (Mazzotti et $a l$., 2009 ; Glen et al., 2007 ; Kpéra, 2003).

Le Burkina Faso possède plusieurs retenues d'eau autour desquelles se sont construits de petits villages. Deux d'entre eux (Bazoulé et Sabou) sont célèbres pour être la terre d'accueil du crocodile du Nil d'Afrique de l'Ouest (C. suchus). Cette espèce est lointainement apparentée mais souvent confondue avec le crocodile du Nil (C. niloticus). Mais des études génétiques ont récemment démontré que ces deux crocodiles sont des espèces distinctes (Hekkala et al., 2011 ; Shirley et al., 2013). Considérées comme des sites naturels sacrés, ces mares ont une importance culturelle, sociale et spirituelle pour les populations. Elles remplissent une fonction vitale pour la conservation des crocodiles et la biodiversité en général et présentent ainsi un intérêt touristique et écologique. Ces mares qui sont des lieux d'offrandes pour demander les faveurs des ancêtres sont également devenues des sites touristiques. Les crocodiles sont intégralement protégés au Burkina Faso à travers la législation forestière nationale (loi 006/97/ADP portant code forestier au Burkina Faso). Ainsi que des dispositions internationales comme la convention de la CITES à laquelle le Burkina Faso a adhéré en 1989. A côté de ces instruments juridiques modernes de protection, certaines dispositions coutumières propres à certaines régions contribuent à la protection des crocodiles. Malgré ces dispositions et réglementations en vigueur pour la protection des crocodiles, plusieurs menaces pourraient peser sur leurs populations. Ce sont, le défrichement incontrôlé de terres cultivables, la pêche anarchique, le surpâturage et d'autres activités anthropiques (Kpéra, 2007 ; Webb et al., 2010).

Toutefois, peu d'études se sont intéressées à cette communauté reptilienne sur ces deux sites sacrés. Les différents travaux effectués sur les crocodiles au Burkina Faso sont pour la plupart des rapports d'activités et ont consisté en des observations ponctuelles et un inventaire superficiel (Bathiono, 2007 ; Nagalo, 2011). Outre ces quelques activités de recherche, on connaît vraiment très peu de choses sur les relations Hommes-crocodiles et 
les mesures de conservation endogènes. Le présent travail a été initié avec pour objectifs de déterminer les perceptions et les croyances des populations sur les crocodiles; de faire l'inventaire de la faune de crocodiles et évaluer l'influence de quelques paramètres anthropiques sur leur abondance.

\section{Méthodologie \\ Sites d'étude}

Deux mares situées en zone rurale ont été retenus pour cette étude. Il s'agit des mares sacrées de Bazoulé et de Sabou.

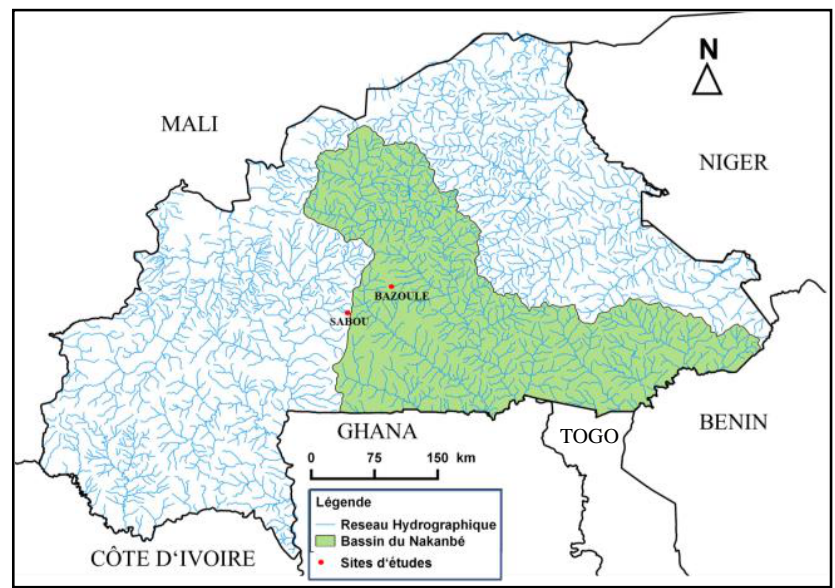

Figure 1 : carte de localisation des sites d'étude

Bazoulé est un village de la commune rurale de Tanghin-Dassouri, province du Kadiogo dans la région du Centre. Le village a une population estimée à 5000 habitants (RGPH, 2006). Il est situé entre la coordonnée $12^{\circ} 19^{\prime} 18^{\prime}$ 'Nord et $1^{\circ} 42^{\prime} 37^{\prime}$ 'Ouest. Le village de Bazoulé possède une mare peuplée de crocodiles sacrés lui conférant un intérêt touristique de grande importance au plan national. La mare est constituée d'un évasement naturel occupant une surface de plus de trois hectares. Elle est alimentée en eau par trois marigots prenant leurs sources dans les villages environnants (Bingo, Tanghin-Dassouri). Des travaux de rénovation ont été engagés en mai 2017 pour augmenter sa capacité. Au plan économique, l'agriculture et l'élevage constituent les principales activités des populations locales. Les habitations sont très dispersées autour de la mare qui se situe au centre du village.

Sabou est une commune rurale de la province du Boulkiemdé située dans la région du Centre Ouest (Koudougou). Elle est située entre $12^{\circ} 04^{\prime} 00^{\prime}$ ' Nord et $2^{\circ} 14^{\prime} 00^{\prime \prime}$ Ouest. La mare aux crocodiles sacrés est située au côté nord de la ville de Sabou à $27 \mathrm{~km}$ au sud de Koudougou et à quelques 80 kilomètres de Ouagadougou sur la route nationale $\mathrm{n}^{\circ} 1$. La commune a une superficie de $449 \mathrm{~km}^{2}$ avec une population estimée à 45877 habitants (RGPH, 2006). La 
mare constitue le principal site touristique de la commune, lui conférant ainsi une réputation nationale.

\section{Collecte de donnée}

\section{Enquêtes auprès des populations}

Un questionnaire semi structuré a été préalablement élaboré à l'aide de consultations documentaires (White et al., 2005 ; Baribeau et al., 2010 ; Shirley et al., 2012) et de personnes ressources. Le questionnaire a été réajusté après une administration préalable à titre d'essai. Il comportait des variables d'identification des enquêtés (nom, prénoms, âge, sexe, profession, village d'origine) et des questions relatives aux activités pratiquées, la perception de la population et leur contribution à la gestion des crocodiles. La population cible était constituée des gestionnaires des mares, des tradi-praticiens, des notables du village, des pêcheurs et d'autres personnes ressources sensées avoir une certaine connaissance sur le crocodile. Ces enquêtes ont été réalisées sous forme d'interviews directes de 15 à 20 minutes et tous les répondants avaient plus de 18 ans. Les enquêtes se sont déroulées dans les habitations situées à proximité de chaque mare. Des guides locaux ont été sollicités pour faciliter la communication auprès de la population. Un total de 59 personnes a été interviewé dans le village de Bazoulé contre 62 personnes dans la commune rurale de Sabou. Le questionnaire adressé aux populations devrait permettre de connaître les perceptions, les raisons et l'origine de la sacralisation des crocodiles. Ainsi que, les relations particulières que ces populations entretiennent avec les crocodiles.

\section{Inventaire des populations de crocodiles}

Pour analyser l'abondance des crocodiles dans les mares sacrées de Bazoulé et de Sabou, deux types d'inventaires ont été realisés : un inventaire diurne et un inventaire nocturne (dénombrement par l'utilisation des projecteurs nocturnes) conformément aux méthodes employées par Bayliss (1987); Santiapillai et al., 2001 ; Shirley et al., (2012) et Fukuda et al., (2013). L'inventaire diurne a consisté à dénombrer les individus de crocodiles en mouvement sur les berges ou dans l'eau. Le dénombrement a été effectué en deux (2) périodes de la journée, d'abord de 7 h00 à $12 \mathrm{~h} 00$ puis de $15 \mathrm{~h} 00$ à $18 \mathrm{~h} 00$. L'inventaire nocturne a été réalisé entre $19 \mathrm{~h} 00$ et $23 \mathrm{~h} 00$. A cette période, des lampes torches ou lampes frontales et des spots lumineux ont été utilisés pour l'éclairage. Pour chaque inventaire, la date, l'heure de début et de la fin et la distance parcourue sont notées. L'inventaire a consisté à faire le tour de chaque mare à pied car ce sont des retenues d'eau de petite taille. Des jumelles ont été utilisées pour faciliter le dénombrement des crocodiles à distance avant qu'ils ne s'immergent. Un appareil photographique a été utilisé pour photographier les individus de crocodiles. Lorsqu'un crocodile a été 
aperçu, il était compté et sa taille était estimée de façon visuelle puis classé selon la structure de classe d'âge standard: les nouveau-nés $(<0,5 \mathrm{~m})$, les juvéniles $(0,5-1,0 \mathrm{~m})$, les sub-adultes $(1-1,50 \mathrm{~m})$ et les adultes $(>1,5 \mathrm{~m})$. Dans le cas où la taille d'un crocodile n'a pas pu être estimée avec précision, l'individu de crocodile était compté et rangé dans la catégorie Yeux seulement (Ys).

Pour chaque site, le nombre d'individu a été estimé en utilisant les principes suivants. La distance parcourue pour chaque mare a été fixée en faisant le pourtour. Ensuite, pour chaque sortie d'inventaire, le nombre individus de crocodiles a été compté par classe d'âge. Le nombre total de crocodiles pour chaque site correspond à la somme des valeurs maximales du nombre d'individu dans chaque classe d'âge pour toutes les sorties.

\section{Caractérisation des paramètres anthropiques}

Afin de caractériser les différents paramètres susceptibles d'influencer l'abondance des crocodiles, une fiche technique a été élaborée. Elle permettait d'enregistrer les informations sur les activités anthropiques grâce à des observations directes sur le terrain à chaque sortie. Lors des observations des informations sur la présence / absence de : tourisme, habitations, pâturage, maraîchage, traces de braconnage, lessive et la pêche sont enregistrées. Les données ont été quantifiées en attribuant une valeur numérique de $0,1,2$ ou 3 selon le degré de chaque paramètre ou variable considéré. $0=$ si le paramètre n'existe pas ; $1=$ si le paramètre faible $; 2=$ si le paramètre est moyen et $3=$ si le paramètre est abondant.

\section{Analyse des données}

Les données collectées au cours de l'enquête ethno-zoologique ont été enregistrées sur une fiche de collecte, puis transférées dans une feuille de Microsoft Excel version 2016. Ces données ont été représentées sous forme d'histogrammes à l'aide du Microsoft Excel version 2016. Afin d'analyser l'influence des différentes variables anthropiques sur l'abondance des populations de crocodiles, les données de suivi sur les abondances ont été croisées avec les variables anthropiques grâce à une analyse canonique. Le logiciel CANOCO (Canonical Ordination of the Communities) pour Windows version 4.5 a été utilisé pour cette analyse.

\section{Résultats}

\section{Origine et historique du crocodile de Bazoulé et de Sabou}

À Bazoulé comme à Sabou l'anthropomorphisme est une chose courante. Toutes les ethnies qui y vivent s'interdisent de tuer ou de consommer le crocodile. Selon les guides Prospère Kaboré, René Kaboré et les notables 
des deux villages, l’origine du crocodile sacré remonte vers les années 1400 . Mais chaque village a sa propre histoire.

À Bazoulé le crocodile a été considéré comme un animal sacré depuis le règne de leur ancêtre Naba Kouda vers les années 1400. À cette époque il y avait une grande sécheresse et la population souffrait énormément par manque d'eau. Pour s'approvisionner en eau il fallait parcourir plus de 15 kilomètres dans les villages environnants comme Oueglega, Doudoulma et Nabitenga. Les crocodiles sont apparus après une grande pluie. Ils avaient creusé un grand trou sous les buissons pour y faire leurs nids. À partir de cet instant, il ne manquait plus d'eau à ce niveau dans le village et la population s'approvisionnait dans ce trou qui deviendra plus tard la mare aux crocodiles de Bazoulé. En guise de reconnaissance, le village organisa une grande fête. Jusqu'à nos jours, le Googhe Naba est chargé de l'organisation la fête coutumière annuelle appelée Koom-lakré en langue Mooré qui signifie « vaincre la famine ». Cette cérémonie qui est dédiée aux crocodiles est organisée tous les derniers dimanches du mois d'octobre de chaque année. À cette occasion des sacrifices sont offerts comme, un coq, un bouc et un âne. Selon le chef de Bazoulé, les crocodiles sont les protecteurs du village. Ils ont le pouvoir d'exaucer des vœux. Ils peuvent aussi prédire le décès d'un notable du village par plusieurs signes tels que les cris déchirants pendant la nuit autour des concessions. Quand un crocodile meurt, il est enterré comme un humain tout en respectant tous les rites possibles. Selon le chef du village, le plus vieux crocodile avait 95 ans et il est mort le 05 août 2005. Ce jour-là une grande pluie s'était abattue sur tout le village tout au long de l'enterrement du crocodile.

Quant aux crocodiles de la mare de Sabou, selon les informations recueillies auprès des anciens, notables et guides, l'histoire de ces reptiles sacrés est liée à l'histoire d'un chasseur de Sabou qui a été sauvé par un crocodile vers les années 1450. L'ancêtre des habitants de Sabou, qui était un chasseur, s'était perdu en brousse sans aucune source d'approvisionnement en eau. Assoiffé et affamé, au bord de la mort, un crocodile est venu lui apporter quelques gouttes d'eau provenant de sa longue queue restée humide. De gratitude, le chasseur lui jura protection. Depuis ce temps, les crocodiles sont sacrés à Sabou et ils vivent en harmonie avec la population. Ils représentent les âmes des descendants de la famille Kaboré à Sabou selon Kuilga Kaboré, guide depuis 1989. Au départ, les crocodiles ne vivaient pas dans la mare construite à la main. C'est en réalité au fil du temps qu'ils ont été transportés dans la mare actuelle et ils incarnent les âmes des habitants des quartiers dorés et Tensomb'Yiri, situés à côté de la mare. Des sacrifices de poulets sont offerts annuellement aux crocodiles par les notables du village. Lorsqu'un crocodile meurt, il est enterré comme un être humain dans le cimetière dédié aux crocodiles. 


\section{Statut sacré et mesures de conservation locale}

$\mathrm{Au}$ total, 59 personnes ont été interviewées à Bazoulé soit $72 \%$ d'hommes et $28 \%$ de femmes contre 62 personnes à Sabou soit $73 \%$ d'hommes et $27 \%$ des femmes. Tous les enquêtés, hommes comme femmes, reconnaissent le statut sacré des crocodiles qui vivent dans les deux mares sacrées. Les crocodiles sont protégés de la chasse avec une interdiction formelle de ramasser les œufs ou de consommer la viande à Bazoulé comme à Sabou. Les crocodiles ont été incorporés dans les croyances et les coutumes dans ces deux villages. Les populations ont dans leur histoire accordée une importance primordiale dans les croyances païennes aux crocodiles. Les crocodiles sont protégés dans le cercle de la tribu des Kaboré dans ces villages.

Le Naaba (chef coutumier) assure avec le soutien de sa communauté l'entretien et la conservation des rites culturels à Bazoulé. Pour cela, une structure coutumière existe avec des notabilités ayant des responsabilités précises, chargées de coordonner et d'organiser de façon pratique le bon déroulement des activités de conservation de la mare. L'Association pour le Développement Touristique de Bazoulé (ADTB) regroupant la jeunesse de Bazoulé assure la gestion pratique et la protection des crocodiles à Bazoulé. La mare de Sabou est gérée par une structure privée et aidée par l'Association des Amis de Sabou.

\section{Relations Hommes-crocodiles et mesures de protection endogènes}

Les crocodiles, anormalement inoffensifs sont des animaux crépusculaires. À Bazoulé comme à Sabou, le crocodile est considéré comme un protecteur et donc vénéré par plusieurs tribus de chaque localité. Ils profitent de la tombée de la nuit pour sortir de la mare et venir jusque devant les concessions soit pour chercher à manger, soit pour faire leur nid ou parfois pour annoncer une triste nouvelle qui arrive dans le village. Compte tenu de leur caractère inoffensif, ces reptiles bénéficient d'une bonne protection de la population. Les conflits Homme-crocodiles sont très rares dans ces deux villages. Selon les résultats des enquêtes, plus de $97 \%$ des personnes interviewées sont unanimes qu'aucun être humain n'a été tué par un crocodile dans les deux villages depuis plus de 80 ans. La population (25\%) estime que lorsqu'il y a une attaque ou une morsure d'un humain par un crocodile, cela relève d'une punition divine des ancêtres. Mais, que ce cas n'a jamais été enregistré dans ces villages. Les seuls cas de conflits qui existent $(10 \%$ à Bazoulé et $30 \%$ à Sabou) sont les captures d'animaux domestiques qui pâturent et s'abreuvent sur le lit des mares. Les crocodiles femelles sont réputés être les plus agressives surtout en période de ponte. Les crocodiles sont adorés par la population et chaque année, une fête coutumière est organisée dans chaque village en guise de reconnaissance et de remerciement aux 
crocodiles (figure 2). A l'occasion de cette cérémonie des poulets, des cabris, du dolo (boisson locale) et des mets sont offert comme sacrifice.
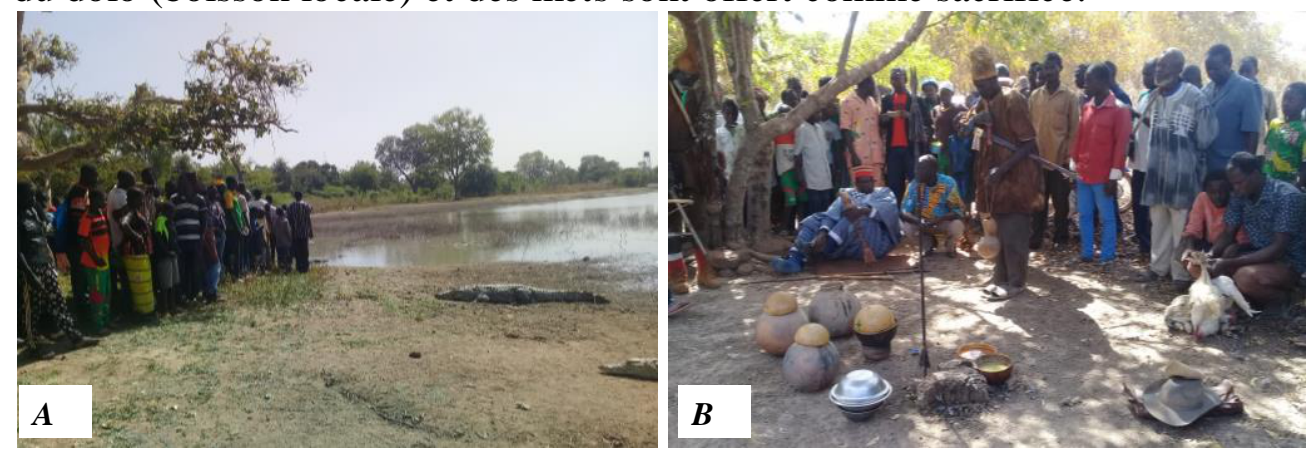

Figure 2 : la fête coutumière à Bazoulé ou le « koom-lakré » dédiée aux crocodiles a : observation des crocodiles par la population ;

$\mathrm{b}$ : les offrandes composées de poulets, des cabris, de boisson et des mets locaux

\section{Estimation de la taille des populations de crocodiles}

Un effectif total de 268 individus de crocodiles a été dénombré à Bazoulé sur une distance totale de 3,50 km parcourue soit un taux de rencontre de 76,57 crocodiles $/ \mathrm{km}$. À Sabou sur une distance totale de 4,6 km parcourue, 249 crocodiles ont été dénombrés soit un taux de rencontre de 54,13 crocodiles $/ \mathrm{km}$. En termes d'abondance relative, les nouveau-nés $(25 \%$ à Bazoulé contre $22 \%$ à Sabou) et les juvéniles (17\% à Bazoulé contre $22 \%$ à Sabou) sont plus abondants que les adultes et subadultes respectivement de $10 \%$ et $12 \%$ à Bazoulé contre $14 \%$ et $12 \%$ à Sabou (figure 3). Les individus de crocodiles classés «Yeux seulement » ne sont pas représentés. Les nouveaunés $(25 \%)$ dominent le peuplement à Bazoulé tandis que les adultes sont moins observés. A sabou, ce sont les juvéniles (22\%) qui dominent.
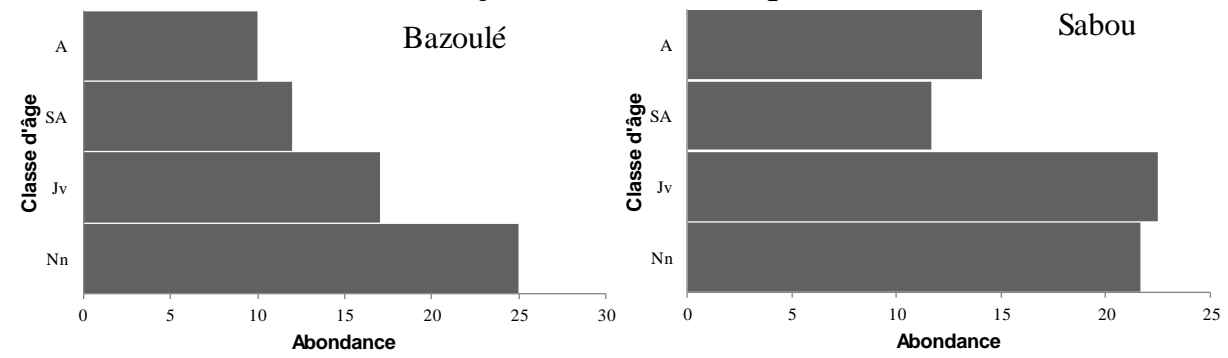

Figure 3 : structure de la population de crocodiles à Bazoulé et à Sabou $\mathrm{A}=$ adultes $; \mathrm{SA}=$ subadultes $; \mathrm{Jv}=$ juvéniles $; \mathrm{Nn}=$ nouveaux nés ;

Le nombre de crocodiles rencontré avant cette étude dans les mares a été estimé entre 85 et 120 individus en 2006 par la population enquêtée à Bazoulé et 75 à 100 individus à Sabou. Alors que maintenant cette taille connait une augmentation et a été estimée à 268 individus à Bazoulé contre 249 à Sabou. Plus de 98\% des personnes enquêtées s'accordent à dire que le 
nombre de crocodile a connu une augmentation dans ces mares sacrées. Les raisons de cette augmentation ont été évoquées par les populations enquêtées. A cet effet, deux (2) raisons principales ont été révélées par les enquêtés (figure 4). La raison totémique est l'une des raisons dont la fréquence de mention s'élève à $80 \% \%$ à Bazoulé contre $58 \%$ à Sabou. La seconde raison qui a été aussi évoqué par la population est la protection et l'interdiction de tuer les crocodiles (17 \% à Bazoulé contre $32 \%$ à Sabou)

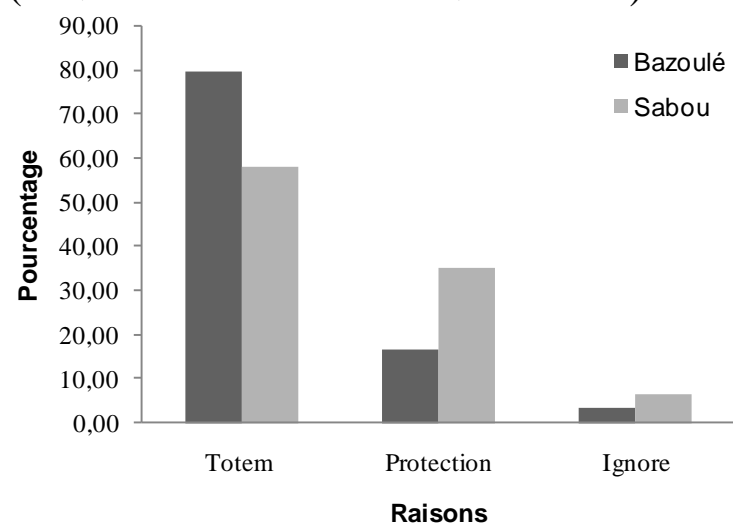

Figure 4 : Raisons de l'abondance des crocodiles dans ces mares sacrée

\section{Importance socio-économique et culturelle}

A Bazoulé comme à Sabou, les crocodiles jouent d'importants rôles dans divers domaines par leur présence dans les mares. Les plus importantes sont le rôle économique, socio-culturelle et religieux. Ainsi, ils attirent chaque jour des touristes au niveau national et international par leur présence. Un flux touristique important a été noté à Bazoulé, avec une moyenne de 2553 visiteurs en 2016 contre 2738 en 2017 (données personnelles).

A Sabou le nombre de touriste a été estimé à 2320 visiteurs au cours de l'année 2016. Les crocodiles jouent également un rôle de protection divine de la population autochtone de Bazoulé et de Sabou. Les crocodiles sont sollicités chaque année par les populations qui viennent leur demander la santé, la fertilité, la réussite, la fortune et la protection contre les ennemis selon le chef de Bazoulé. Le rôle de protection a été le service le plus cité par la population (81,8 \%) à Bazoulé contre 74,6\% à Sabou (figure 5). Ensuite vient le rôle touristique $(25 \%)$ à Bazoulé et $(19 \%)$ à Sabou. L'éducation environnementale est le service le moins cité par la population $(0,6 \%)$ à contre $(0,3 \%)$ à Sabou. Vue l'importance des reptiles, plus de $65 \%$ des personnes interrogées se disent très satisfaites et ont une bonne perception de ces crocodiles sacrés. 


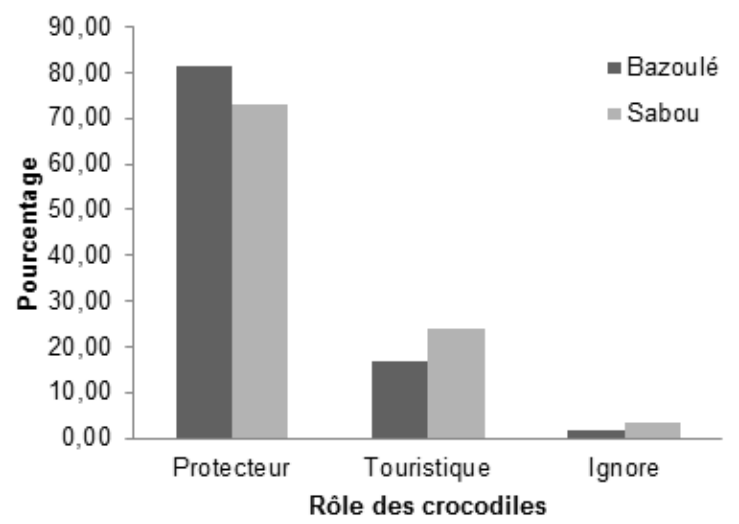

Figure 5 : perception de l'importance des crocodiles à Bazoulé et Sabou

\section{Impact des activités anthropiques sur l'abondance des crocodiles}

La figure 6 présente les résultats de l'ordination de l'abondance des individus de crocodiles par classe d'âge et des variables anthropiques à Bazoulé. L'analyse en composantes principales de la matrice abondance montre que l'axe 1 qui extrait $81,3 \%$ de la variance, on note une corrélation positive entre les nouveau-nés et la confection des briques traditionnelles alors qu'ils sont négativement corrélés au maraîchage. En revanche, cette classe d'âge est négativement corrélée au prélèvement de la végétation. Sur l'axe 2, qui extrait 90,6\% de la variance, il existe une corrélation entre l'activité de pêche et l'abondance des crocodiles adultes. Les adultes et les juvéniles sont négativement corrélés au braconnage.

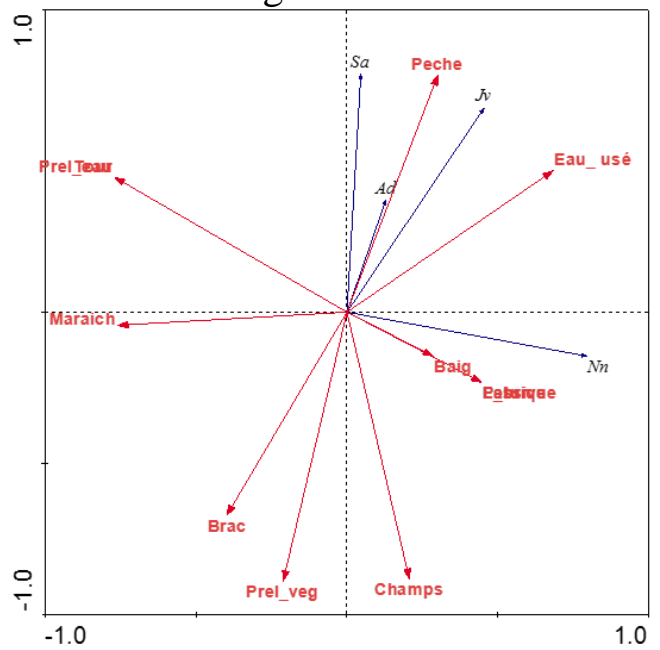

Figure 6 : Résultats de l'Analyse de Redondance des abondances des crocodiles (en noir) et des variables anthropiques (en rouge) à Bazoulé

Nn= nouveau-nés ; Jv = juvéniles ; Sa= subadultes ; Ad= adultes.

Eau_us : eau usées ; Pche: pêche; Tour : tourisme, patur : pâturage ; maraich : maraîchage ; prel_veg: prélèvement de la végétation ; baig :baignade ; brac : braconnage ; F_brique : fabrication des briques 
La figure 7 présente l'ordination des abondances des classes d'âges et les variables anthropiques à Sabou. L'analyse en composantes principales de la matrice regroupant les variables anthropiques et l'abondance des crocodiles montre que : sur l'axe 1 qui extrait $60,8 \%$ de la variance, on observe un regroupement de paramètre comme le tourisme, les champs, la baignade. Ces variables présentent une corrélation négative avec l'abondance des nouveaunés. Sur l'axe 2 qui extrait $59,2 \%$ de la variance, on note une corrélation positive entre la confection des briques traditionnelles, le prélèvement de la végétation et les crocodiles adultes.

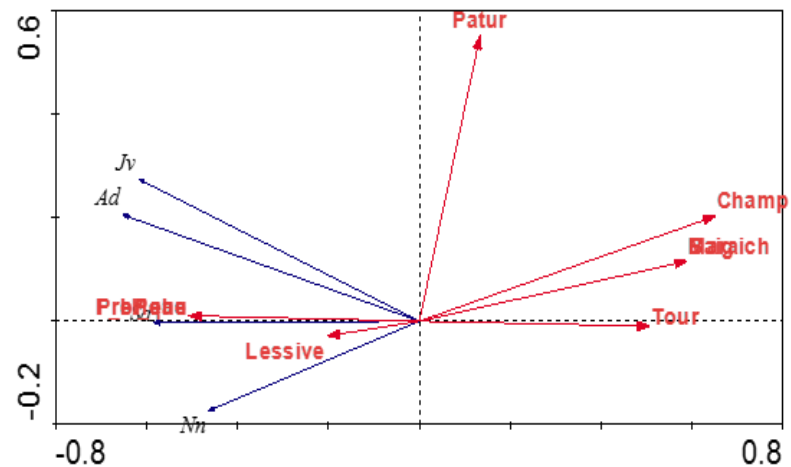

Figure 7 : Résultats de l'Analyse de Redondance des abondances des crocodiles (en noir) et des variables anthropiques (en rouge) à Sabou

Nn= nouveau-nés $; J v=$ juvéniles $;$ Sa $=$ subadultes $;$ Ad $=$ adultes.

Pche: pêche; Tour : tourisme, patur : pâturage ; maraich : maraîchage ; baig :baignade ; brac : braconnage; F_brique : fabrication des briques; prel_eau : prélèvement d'eau

\section{Discussion}

Le crocodile tient une place considérable dans la culture et les coutumes des populations de Bazoulé et celle de Sabou. Une attention particulière est accordée à ces reptiles dans ces deux villages en raison de leur importance dans la société. Les résultats des enquêtes ont permis de connaître les perceptions locales que les habitants ont des crocodiles. L'origine et l'histoire du crocodile sacré diffère d'un village à l'autre. Ce qui est tout à fait raisonnable car chaque communauté possède son histoire et sa propre culture. Mais le point commun qui a permis d'apprivoiser $C$. suchus dans ces deux localités demeure le rôle joué pour sauver l'ancêtre de chacune des deux populations de la soif. En guise de récompense, des sacrifices sont offerts chaque année aux crocodiles sacrés. Cela permet de maintenir le statut sacré et d'éviter des conflits entre Homme-crocodile. Les considérations totémiques et les perceptions locales constituent un atout majeur dans la protection et la conservation du crocodile. Ce qui a contribué à une augmentation de leur population. Selon (Brown et al.,2004 et Aust et al., 2009), certaines mesures de conservations ont permis d'empêcher la chasse et l'abattage clandestin de crocodiles dans certaines régions du monde. Ce qui a entrainé une 
augmentation de la population de crocodile dans ces régions. Par contre dans les régions où les crocodiles sont perçus comme un danger pour l'Homme et le bétail, ils ont été délibérément exterminés (Santiapillai et al., 2001). Les crocodiles représentent pour les populations une valeur traditionnelle dont les villageois de toutes catégories (les autochtones, et les étrangers) connaissent bien son importance. Ils incarnent les aïeux, et au-delà des limites des deux villages, les villages voisins semblent avoir conscience de l'intérêt accordé aux crocodiles. La protection, le développement du tourisme, ainsi que l'augmentation de la capacité des mares suites aux travaux de rénovation permettant de stocker une importante quantité d'eau sont des facteurs qui expliqueraient aussi l'accroissement des populations de crocodile sacrés.

Ces mesures de conservation ont permis de maintenir de très bon rapport entre Homme-crocodile. Les personnes interrogées sont unanimes qu'aucun être humain n'a été attaqué par un crocodile sur ces sites. Cela pourrait s'expliquer par le fait que les crocodiles sont apprivoisés et bénéficient des protections de toute la population donc ils ne se sentent pas menacés. En plus des poulets sont offert aux crocodiles grâce à la fréquentation touristique. Chaque visiteur a l'obligation de payer un poulet qui servira comme appât pour faire sortir un nombre important de crocodile hors de l'eau. Les seuls cas de conflit concernent les attaques des animaux domestiques qui divaguent sur les rives des mares. Selon (Schmitz et al., 2004), C. suchus a cette particularité d'attaquer beaucoup plus de bétail que des humains. Pour Lamarque et al., 2009, les conflits entre Hommes et animaux sauvages existent depuis longtemps et concernent tous les continents y compris l'Afrique où vivent de grands mammifères herbivores comme les éléphants et les grands carnivores comme les lions et surtout les crocodiles.

L'augmentation de la population de crocodiles pourrait s'expliquer par le statut sacré dont ils bénéficient. Par ce statut sacré, il est strictement interdit de tuer le crocodile, de ramasser ces œufs ou de consommer sa viande. Toute personne qui tuerait un crocodile doit être dénoncée et puni selon la tradition. Ce qui a favorisé des taux de rencontres important dans ces mares $(76,57$ crocodiles/km à Bazoulé contre 54,13 crocodiles/km à Sabou). Ces densités sont très importantes par rapport aux travaux de Sai et al., (2016) ; Botha et al., (2011). En effet Sai et al., (2016), avaient rencontré 82 crocodiles du Nil avec un taux de rencontre de 2,28 crocodiles/ $\mathrm{km}$ dans les rivières de Kove et Sengwa au nord-ouest du Zimbabwe. Alors que Botha et al., (2011) ont rencontrés un total de 8 crocodiles dans le barrage de Loskop en Afrique du Sud au cours de leurs travaux avec un taux de rencontre de 0,11 crocodiles / $\mathrm{km}$. Le nombre élevé de nouveau-nés pourrait s'expliquer par la période de suivi (mai-juin). Cette période de suivis coïncide avec le début de la saison des pluies et cela corresponde à la période d'éclosion des crocodiles. La faible densité des subadultes et des adultes pourrait s'expliquer par le fait que ces 
derniers sont attentifs au moindre bruit donc disparaissent rapidement sous l'eau. Cela confirme la théorie de Hutton et Woolhouse, (1989), qui estimaient que les animaux adultes sont plus mobiles et s'immergent rapidement sous l'effet de la lumière.

Le crocodile a une valeur économique et culturelle indéniable au plan national en général et sur les sites sacrés en particulier. Le crocodile joue un rôle de protection divine et culturel à Bazoulé et à Sabou. Cela est en accord avec les travaux de (Kpéra, 2002 ; 2003) au Benin qui avaient montré que les crocodiles sont très importants pour les communautés locales, en raison de leur utilisation dans la médecine traditionnelle et les considérations spirituelles et totémiques locales. Le flux important de touristes enregistré engendre des devises pour ces villages. Ces devises issues de ces visites servent au développement local du village. Pour Telleria et al., (2008), le crocodile a une valeur touristique indéniable.

Bien que les croyances traditionnelles confèrent à $C$. suchus une certaine protection, les activités anthropiques pourraient avoir un impact pour sa conservation. Treves, et al., (2009) ont affirmé que la population de crocodile a fortement diminué dans le monde du fait de l'accroissement des activités anthropiques. La corrélation négative entre l'abondance des nouveaunés et le maraîchage traduit le fait que la pratique des activités maraichères entraine des effets néfastes sur cette catégorie d'individu de crocodile. Des nids de crocodiles sont parfois détruit sur le lit des mares à cause de cette activité. Selon Kpéra (2002 ; 2007), les activités anthropiques aux niveaux des mares aux crocodiles du Bénin, seraient des facteurs qui influenceraient négativement la vie de ces populations. Les travaux champêtres réduisent les lieux de ponte du crocodile et augmentent la pollution agricole par l'utilisation abusive des pesticides ce qui n'est pas sans conséquence sur la vie des reptiles (Kpéra et $a l ., 2007$; Webb et $a l ., 2010)$. La corrélation positive qui existe entre la pêche et l'abondance des crocodiles adultes s'explique par le fait que les crocodiles adultes prélèvent les poissons piégés par les filets des pêcheurs pendant la nuit. Cela est contradictoire avec les travaux de McGregor, (2005), qui avaient estimé que l'activité de pêche entraîne souvent la mort ou de sévères dommages sur les crocodiles surtout les juvéniles, qui sont capturé de façon accidentelle dans les filets de pêche. La mare de Sabou présente les mêmes pressions anthropiques qu'à Bazoulé. Des corrélations négatives sont observées entre les nouveau-nés et les paramètres comme la baignade, le tourisme et les champs. En effet comme l'avait souligné Kpéra et al., 2007, les pratiques agricoles réduisent les lieux de ponte du crocodile et augmentent la pollution. Aussi lors des activités de baignade, la tranquillité des crocodiles est dérangée. Selon Botha et al., (2011) ; Fukuda et al., (2014), les différentes activités anthropiques influencent la distribution des crocodiles. 


\section{Conclusion}

La présente étude a permis de connaître l'origine et l'historique des crocodiles sacrés des mares de Bazoulé et de Sabou et de recenser les perceptions des populations. La sacralisation des crocodiles remonte depuis des siècles et est basée sur des fondements culturels de chaque localité. L'étude a montré que les crocodiles sont très importants pour la population locale. Compte tenu de l'importance rôle que jouent les crocodiles, les populations se disent très satisfait par leur présence dans les mares. Bien que les crocodiles bénéficient d'une protection culturelle et religieuse, ils subissent de nos jours de fortes pressions dues aux activités anthropiques pratiquées autour des mares. Malgré ces menaces anthropiques, les mares et les crocodiles sacrés de Bazoulé et de Sabou constituent de véritables sites d'attraction touristique. Ainsi, la valeur culturelle du crocodile sacré vire sensiblement vers une valeur économique. Pour une bonne protection et une gestion durable des mares et des crocodiles sacrés, des séances de sensibilisation des populations et une formation des guides sur la gestion des sites devraient être entreprises par les autorités.

\section{References:}

1. Aust P., Boyle B., Fergusson R., \& Coulson T., (2009). The impact of Nile crocodiles on rural livelihoods in north eastern Namibia. S.A Journal of Wildlife Research 39(1): 57- 69.

2. Baribeau C. et Germain M., (2010). L'entretien de groupe : considérations théoriques et méthodologiques, Recherches Qualitatives, Vol 29 (1), 28-49.

3. Bationo, Y., (2007). Les Crocodiles du Burkina Faso : Diagnostic situationnel et perspectives Communication au $1^{\mathrm{er}}$ congrès du GSC des pays de l'Afrique de l'Ouest, 11p.

4. Bayliss, P., (1987). Survey Methods and Monitoring within Crocodile Management programmes, In Wildlife Management: Crocodiles and Alligators ed by J.W. Webb, S. Charlie Manolis \& Peter J. Whitehead. Survey Beatty and Sons Pty Limited in association with the Conservation Commission of the Northern Territory, pp. 157-175.

5. Botha H., Van Hoven W., Guillette, LJ., (2011). The decline of the Nile crocodile population in Loskop Dam, Olifants River South Africa. Water SA, 37(1) :103-108. DOI: 10.4314/wsa. v37i1.64109.

6. Brown C. J., Stander P., Meyer-Rust R., Mayes S., (2004). Results of a Nile Crocodile (C. niloticus) survey in the river systems of Northeast Namibia. IUCN/SSC Crocodile Specialist Group Newsletter. IUCN, Gland, Switzerland, 23 (3) 18-21.

7. Ceballos, G., Ehrlich, P. R., Barnosky, A. D., García, A., Pringle, R. M. and Palmer, T. M., (2015). Accelerated Modern Human-Induced 
Species Losses: Entering the Sixth Mass Extinction. Science Advances 1(5): e1400253.

8. Fukuda Y., Saalfeld K., Webb G., Manolis C., et Risk R., (2013). Standardized methods of spotlight surveys for crocodiles in the tidal rivers of the Northern Territory, Australia. Northern Territory Naturalist 2013(24):14-32.

9. Fukuda, Y., Manolis, C. et Appel, K., (2014) Management of humancrocodile conflict in the Northern Territory, Australia: review of crocodile attacks and removal of problem crocodiles. Journal of Wildlife Management, 78, 1239-1249.

10. Glen, A.S., Dickman, C.R., Soule, M.E., \& Mackey, B.G., (2007). Evaluating the role of the dingo as a trophic regulator in Australian ecosystems. Austral Ecology, 32, 492-501.

11. Hanski I., (2005). Landscape Fragmentation, Biodiversity Loss and the Societal Response. The longterm consequences of our use of natural resources may be surprising and unpleasant, 6(5): 388-392.

12. Hekkala, E., Shirley, M.H., Amato, G., Austin, J.D., Charter, S., Thorbjarnarson, J., Vliet, K.A., Houck, M.L., Desalle, R. et Blum, M.J. (2011). An ancient icon reveals new mysteries: mummy DNA resurrects a cryptic species within the Nile crocodile. Molecular Ecology. DOI: 10.1111/j.1365-294X.2011.05245. x.

13. Hutton J.M., Woolhouse J., (1989). Mark-recapture to assess factors affecting the proportion of a Nile crocodile population seen during spotlight counts at Ngezi, Zimbabwe, and the use of spotlight counts to monitor crocodile abundance. Journal of Applied Ecology 26: 381395. DOI: $10.2307 / 2404068$.

14. Kpéra G. N., (2002). Impact des aménagements d'hydraulique pastorale et des mares sur la reconstitution des populations de crocodiles dans les Communes de Nikki, Kalalé, Sébgana, Kandi, Banikoara, Kérou, Ouassa-Péhunco et Sinendé. Thèse d'Ingénieur Agronome, FSA/UNB, Bénin. 96p.

15. Kpéra G. N., (2003). Notes on crocodiles in Bénin. IUCN/SSC Crocodile Specialist Group Newsletter. IUCN, Gland, Switzerland, 22 (1), 3-4.

16. Kpéra G. N., (2007). Rapport de mission du premier congrès d'Afrique de l'Ouest du groupe des spécialistes en crocodiles sur la conservation et l'élevage des crocodiles 13-15 novembre 2007, Tapoa-Niger. 25p.

17. Kpéra G. N., Sinsin B., et Mensah G. A., (2007). Mesures de conservation endogènes de la faune Sauvage : Cas des crocodiles au Bénin. In Proceeding of the 42- First Regional Workshop on the Management of Protected Area in West Africa. 2003. Parakou, Bénin, pp.405-414. 
18. Krauss J., Bommarco, R., Guardiola, M., Heikkinen, R. K., Helm, A., Kuussaari, M., Lindborg, R., Öckinger, E., Pärtel, M., Pino, J., Pöyry, J., Raatikainen, K. M., Sang, A., Stefanescu, C., Teder, T., Zobel, M. et Steffan-Dewenter, I., (2010). Habitat Fragmentation Causes Immediate and Time-Delayed Biodiversity Loss at Different Trophic Levels. Ecology Letters 13(5): 597-605.

19. Lamarque, F., Anderson, J., Fergusson, R., Lagrange, M., OseiOwusu, Y. et Bakker, L., (2009). Human-Wildlife conflict in Africa, Causes, consequences and management strategies, FAO. Forestry Paper 157, 98p.

20. Mazzotti, F. J., Best, G. R., Brandt, L. A., Cherkiss, M. S., Jeffery, B.M., et Rice, K.G., (2009) Alligators and crocodiles as indicators for restoration of Everglades ecosystems. Ecological Indicators, 9, S137S149.

21. McGregor, J. (2005) Crocodile crimes: people versus wildlife and the politics of postcolonial conservation on Lake Kariba, Zimbabwe. Geoforum, 36, 353-369.

22. Nagalo B.J., (2011). Les Crocodiles de la Forêt Classée et Ranch de Gibier de Nazinga et possibilité de leur valorisation. Mémoire d'Ingénieur en Vulgarisation Agricole 58p.

23. RGPH, (2006). Etat et structure de la population du Burkina Faso, p935.

24. Sai M., Utete B., Chinoitezvi E., Moyo G.H., and Gandiwa E., (2016). A Survey of the Abundance, Population Structure, and Distribution of Nile Crocodiles (Crocodylus Niloticus) Using Day Ground Surveys in Sengwa Wildlife Research Area, Zimbabwe. Herpetological Conservation and Biology 11 :426-433.

25. Santiapillai C. et de Silva M., (2001). Status, distribution and conservation of crocodiles in Sri Lanka. Biological Conservation 97, 305-318.

26. Schmitz, A., Mausfeld, P., Hekkala, E., Shine, T., Nickel, H., Amato, G., and Bohme, W. (2004) Molecular evidence for species level divergence in African Nile Crocodiles C. niloticus. Comptes Rendus Palevol, 3, 177-177.

27. Shirley, M.H., Vliet, K.A., Carr, A.N. and Austin, J.D., (2013). Rigorous approaches to species delimitation have significant implications for African crocodile systematics and conservation. Proceedings of the Royal Society B: Biological Sciences 281(1776): 2013-2483.

28. Shirley, M.H.; Dorazio, M. R.; Abassery, E.; Elhady, A. A.; Mekki, M. S.; and Asran, H.H., (2012). A sampling design and model for estimating abundance of Nile Crocodiles while accounting for 
heterogeneity of detectability of multiple observers. Journal of Wildlife Management 76(5):966-975; 2012; DOI: 10.1002/jwmg.348. 29. Telleria, J. L., Ghaillani, H. E. M., Fernandez- Palacios, J. M., Bartolome, J. and Montiano, E., (2008). Crocodiles C. niloticus as a focal species for conserving water resources in Mauritanian Sahara. Oryx, 42, 292-295.

30. Treves, A., Wallace, R.B., and White, S., (2009). Participatory planning of interventions to mitigate human-wildlife conflicts. Conservation biology, 23(6):1577-1587.

31. Webb G.J.W., Manolis S.C., and Brien M.L., (2010). Saltwater Crocodile C. porosus. Pp. 99-113 in Crocodiles. Status Survey and Conservation Action Plan. Third Edition, ed. by Manolis S.C., and Stevenson C. Crocodile Specialist Group: Darwin. IUCN.

32. White, P. C. L., Jennings, N. V., Renwick, A. R., and Barker, N. H. L., (2005). Questionnaires in Ecology: a review of past use and recommendations for best practice. Journal of Applied Ecology 42(3) :421-430. 